\title{
Regional Lymph Node Ultrasound
}

National Cancer Institute

\section{Source}

National Cancer Institute. Regional Lymph Node Ultrasound. NCI Thesaurus. Code C137926.

An ultrasound of one or more lymph nodes in an anatomical area of interest. 